\title{
Site-selective transient photoluminescence enhancement of impurity-trapped excitons in $\mathrm{NaMgF}_{3}: \mathbf{Y b}^{2+}$
}

\author{
Rosa B. Hughes-Currie, ${ }^{1}$ Pubudu S. Senanayake, ${ }^{1}$ Jon-Paul R. Wells,,${ }^{1}{ }^{*}$ Michael F. Reid, ${ }^{2}$ Giel Berden, ${ }^{3}$ Roger J. Reeves,${ }^{2}$ and \\ Andries Meijerink ${ }^{4}$ \\ ${ }^{1}$ Department of Physics and Astronomy, University of Canterbury, PB 4800, Christchurch 8140, New Zealand \\ ${ }^{2}$ Department of Physics and Astronomy and MacDiarmid Institute for Advanced Materials and Nanotechnology, \\ University of Canterbury, $P B$ 4800, Christchurch 8140, New Zealand \\ ${ }^{3}$ FELIX Free Electron Laser Facility, FOM Institute for Plasmaphysics Rijnhuizen, P.O. Box 1207, 3430 BE, Nieuwegein, The Netherlands \\ ${ }^{4}$ Debye Institute for NanoMaterials Science, University of Utrecht, P.O. Box 80000, TA 3508 Utrecht, The Netherlands
}

(Received 9 April 2013; revised manuscript received 12 June 2013; published 16 September 2013)

\begin{abstract}
The excited-state structure of impurity-trapped excitons are measured in a multisite system. We use a two-color (UV-IR) pulsed photoluminescence enhancement technique, which probes the interlevel transitions and dynamics of impurity-trapped excitons in doped insulating phosphor materials. The technique is applied to $\mathrm{NaMgF}_{3}: \mathrm{Yb}^{2+}$, which exhibits emission from two charge-compensation centers with peaks at $22300 \mathrm{~cm}^{-1}(448 \mathrm{~nm})$ and $24000 \mathrm{~cm}^{-1}(417 \mathrm{~nm})$. The observed photoluminescence enhancement is caused by a combination of intraexcitonic excitation and electron trap liberation. The electron traps are inferred to have a depth of approximately $800 \mathrm{~cm}^{-1}$.
\end{abstract}

DOI: 10.1103/PhysRevB.88.104304

PACS number(s): 76.30.Kg, 71.35.-y, 71.70.Ch

\section{INTRODUCTION}

Impurity-trapped excitons (ITEs) play an important role in the relaxation processes of the highly excited states of lanthanide ions in phosphor materials but their dynamics are not well understood. ITEs are bound electron-hole pairs with the hole localized on an impurity (in this case the $\mathrm{Yb}^{2+}$ ion) and the electron on nearby lattice sites. ${ }^{1}$ The electron has been assumed to be localized on the neighboring cations ${ }^{2}$ but recent $a b$ initio studies of ITE states have shown that the exciton radius may be smaller. ${ }^{3,4}$

Many materials have ITE states that play a role in relaxation processes but studying impurity-trapped excitons in optical materials is often difficult since the ITEs that form in these systems decay predominantly nonradiatively and so have to be studied indirectly. This means that their formation and nature are still largely unknown. However, there are some systems for which the photoluminescence appears to come directly from ITE states. In particular, certain fluoride crystals doped with $\mathrm{Yb}^{2+}$ are observed to have wide emission bands and large Stokes shifts compared to $4 f^{13} 5 d \rightarrow 4 f^{14}$ transitions, suggesting the emission is from impurity-trapped excitons, which are being formed on the ytterbium ions. ${ }^{2}$ Two-color spectroscopy can reveal details of the energy level structure and dynamical behavior of the excitons formed in these materials. In this paper a site-selective transient photoluminescence enhancement (SSTPE) technique is applied to $\mathrm{NaMgF}_{3}: \mathrm{Yb}^{2+}$ to study impurity-trapped excitons.

SSTPE is a pulsed, two-color excitation technique, which is schematically depicted in Fig. 1. The sample is excited with a UV excitation pulse (in this case $300 \mathrm{~nm}$ ) that resonantly excites the interconfigurational $\mathrm{Yb}^{2+} 4 f^{14} \rightarrow 4 f^{13} 5 d$ absorption. Subsequent decay then populates the ground state of the impurity-trapped exciton. After a time delay of the order of $100 \mu$ s the sample is probed with an IR pulse generated by a free electron laser. The IR pulse excites the impurity-trapped exciton from its ground state (state 2 in Fig. 1) into a higher-energy state (state 4) from which it quickly decays into another emitting excited state with a shorter lifetime than state 2 (state 3 ). As a consequence of the different radiative relaxation rates of the excitonic levels 2 and 3, a temporal enhancement in emission can be detected. The fact that the radiative rate of state 3 is greater than that of state 2 is known from measurements of the temperature dependence of the total radiative lifetime of the system after UV excitation. ${ }^{5}$

The emission band from $\mathrm{NaMgF}_{3}: \mathrm{Yb}^{2+}$ is very wide and the Stokes shift is large so the emission is unlikely be from a $4 f^{13} 5 d \rightarrow 4 f^{14}$ transition. The Stokes shift for $\mathrm{NaMgF}_{3}: \mathrm{Yb}^{2+}$ is $7000 \mathrm{~cm}^{-1}$. This large shift is typical of the emission from an ITE. In $\mathrm{NaMgF}_{3}$ the energy of the spin-forbidden $\mathrm{Yb}^{2+}$ emission deviates from the expected value (based on the spin-allowed emission of $\mathrm{Eu}^{2+}$ ) by $0.44 \mathrm{eV}$, which means the emission is likely to involve conduction band states, indicating ITE emission. ${ }^{2}$

A two-color experimental technique has been previously employed to probe the ITE energy levels of ytterbium-doped calcium fluoride and strontium fluoride ${ }^{6}$ as well as electron trap liberation in ytterbium-doped magnesium fluoride. ${ }^{7}$ The work in this paper expands on these previous studies by presenting a comprehensive model of the temporal dynamics of a multicenter material. The value to this temporal analysis is to provide rate parameters which characterise the ITE and can be used as a basis for similar studies in other materials.

There is current interest in dosimetry and storage phosphor applications of $\mathrm{NaMgF}_{3}$ doped with $\mathrm{Ce}^{3+}$ and divalent Eu and Mn. ${ }^{8-11}$ This study of $\mathrm{NaMgF}_{3}: \mathrm{Yb}^{2+}$ gives details of electron traps relevant to dosimetry applications and could be applied to $\mathrm{NaMgF}_{3}$ doped with other divalent lanthanides.

$\mathrm{NaMgF}_{3}$ has an orthorhombically distorted perovskite structure. ${ }^{12}$ The $\mathrm{Yb}^{2+}$ replace $\mathrm{Na}^{+}$ions. It is likely that there are many charge-compensation centers for the $\mathrm{Yb}^{2+}$ ion, as there are many charge-compensation centers in $\mathrm{KMgF}_{3}$ doped with divalent lanthanides. Excitation spectra of $\mathrm{KMgF}_{3}: \mathrm{Sm}^{2+}$ 


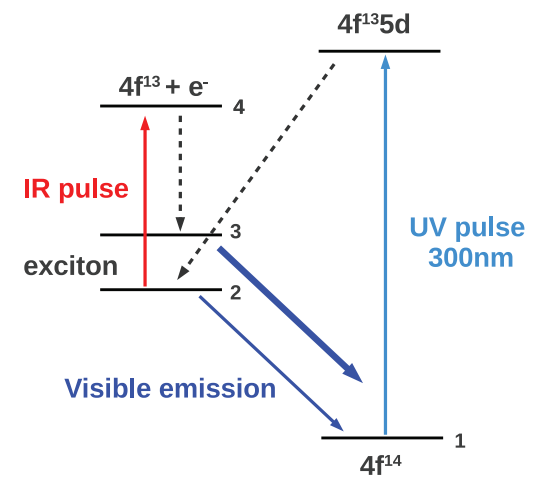

FIG. 1. (Color online) A schematic of the SSTPE method. UV excitation promotes $\mathrm{Yb}^{2+}$ ions from the $\mathrm{Yb}^{2+}$ ground state to a higher $\mathrm{Yb}^{2+}$ state from which they relax nonradiatively into the exciton ground state. There are two primary emitting exciton levels, the ground exciton state (state 2 ) and a higher-energy exciton state (state 3 ). The higher-energy exciton state has a larger radiative rate. The IR pulse promotes excitons from state 2 into higher exciton energy levels, whereby they radiatively relax to the $\mathrm{Yb}^{2+}$ ground state.

indicates five charge comensation centers ${ }^{13}$ and pressuredependant luminescence of $\mathrm{KMgF}_{3}: \mathrm{Eu}^{2+}$ also shows over five charge compensation sites. ${ }^{14}$ Multiple sites are also seen in $\mathrm{NaMgF}_{3}: \mathrm{Sm}^{2+}$. The emission spectrum of $\mathrm{NaMgF}_{3}: \mathrm{Sm}^{2+}$ is much simpler than that of $\mathrm{KMgF}_{3}: \mathrm{Sm}^{2+}$, which indicates divalent lanthanide centers in $\mathrm{NaMgF}_{3}$ have lower symmetry than in $\mathrm{KMgF}_{3} .{ }^{15}$

The emission band of $\mathrm{NaMgF}_{3}: \mathrm{Yb}^{2+}$ is reported to be a convolution of emission from impurity-trapped exciton states of two of the charge-compensating arrangements. ${ }^{2,5}$ The evidence of two emitting sites given by Lizzo et al. ${ }^{5}$ is that the emission peak shape changes with temperature; the excitation spectra and the temperature dependence of the emission decay lifetime is significantly different when the emission is monitored on either side of the band.

\section{EXPERIMENT}

The crystal used in this experiment contained $0.6 \mathrm{~mol} \%$ of ytterbium and was grown using the Bridgman technique in a reducing atmosphere. Ultraviolet excitation at $300 \mathrm{~nm}$ was provided by a frequency doubled traveling wave optical parametric amplifier (OPA, LightConversion Ltd) having a repetition rate of $1 \mathrm{kHz}$ and a pulse length of $3 \mathrm{ps}$. The Dutch free-electron laser (FELIX) in Nieuwegein was used to excite the samples in the infrared. The output of FELIX consists of a $6 \mu$ s long macropulse with a repetition rate of $10 \mathrm{~Hz}$. The IR radiation was tuned between 5 and $25 \mu \mathrm{m}$. The two lasers were synchronized and have an electronically variable delay, which was limited to less than one millisecond due to the repetition rate of the OPA. The two beams were spatially overlapped on the sample with a spot size of approximately $100 \mu \mathrm{m}$. The resultant fluorescence was collected using a TRIAX 320 spectrometer and detected with an RCA C31034 photomultiplier tube. This PMT responds well over the entire range for which emission was measured. The sample was cooled to cryogenic temperatures in a temperature variable Oxford Instruments Microstat optical access cryostat.
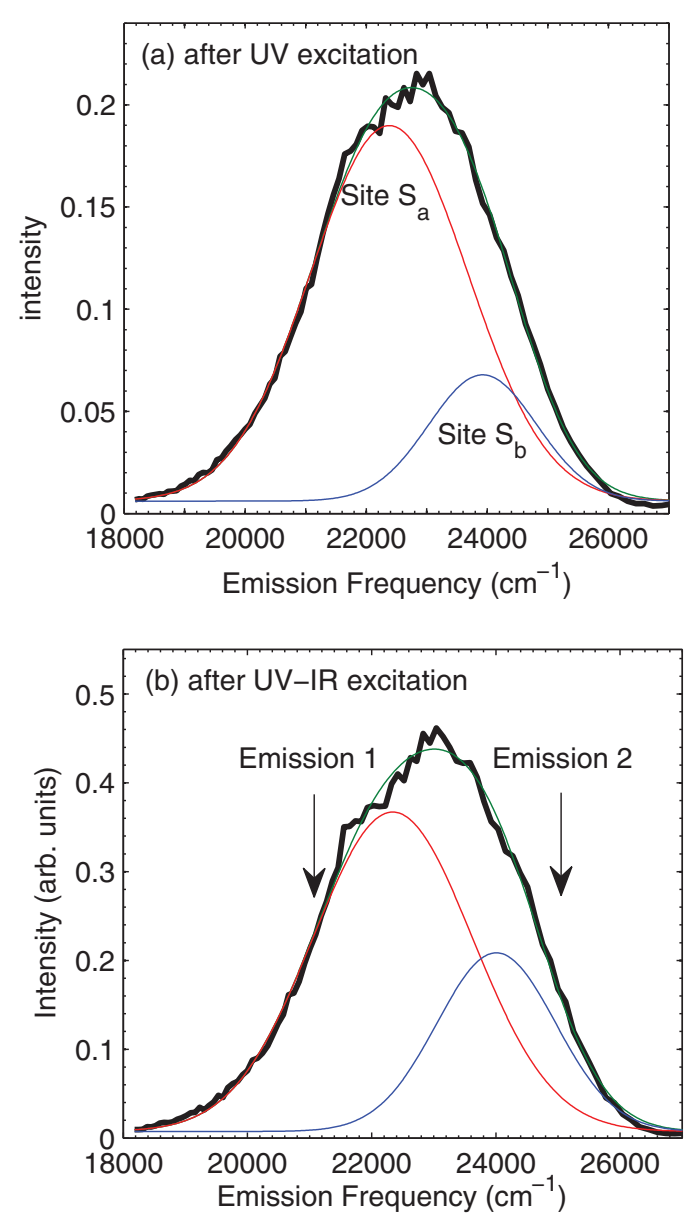

FIG. 2. (Color online) The $8 \mathrm{~K}$ emission spectrum of $\mathrm{NaMgF}_{3}: \mathrm{Yb}^{2+}$ (a) averaged over $20 \mu$ s after UV excitation at $300 \mathrm{~nm}$ and before IR excitation. The emission is modeled as coming from two charge-compensation arrangements with emission bands centered at $22400 \mathrm{~cm}^{-1}$ and $24000 \mathrm{~cm}^{-1}$. (b) averaged over $20 \mu$ s after UV excitation at $300 \mathrm{~nm}$ and IR excitation at $962 \mathrm{~cm}^{-1}$. After excitation with the IR pulse the relative intensity of center $S_{b}$ compared to center $\mathrm{S}_{\mathrm{a}}$ has increased. At $21000 \mathrm{~cm}^{-1}$ (emission 1) practically all the light is from center $S_{a}$, but at $25000 \mathrm{~cm}^{-1}$ (emission 2) the ratio of intensity from centers 1 and 2 is approximately 1:2.

\section{RESULTS}

\section{A. Emission enhancement}

Figure 2 shows the emission spectrum of $\mathrm{NaMgF}_{3}: \mathrm{Yb}^{2+}$ after UV excitation at $300 \mathrm{~nm}$. The observed band is the convolution of emission from two charge-compensation centers, which have frequencies of $22400 \mathrm{~cm}^{-1}(446 \mathrm{~nm})$ and $23900 \mathrm{~cm}^{-1}(418 \mathrm{~nm})$. The two charge-compensation arrangements (arbitrarily labeled center $S_{a}$ and center $S_{b}$ ) can be spectrally deconvolved by monitoring the emission at points on either side of the overall emission band shown in Fig. 2. At $21000 \mathrm{~cm}^{-1}(476 \mathrm{~nm}$, labeled emission 1) the emission is entirely from center $S_{a}$ and at $25000 \mathrm{~cm}^{-1}(400 \mathrm{~nm}$, labeled emission 2) the emission is predominantly from center $S_{b}$. Gaussian profile line fits were applied to the spectra in Fig 2 to obtain the parameters listed in Table I.

Figure 2(a) shows the emission from both centers after UV excitation. It is important to note that temporal analysis in 
TABLE I. Parameters for Gaussian function fits of the emission bands in Fig. 2 for (a) UV excitation only and (b) UV-IR excitation. $\Delta R$ is the change in bond length associated with the transition. ZPL is the zero phonon line, found by adding the Stokes shift (calculated from the width of the band) to the center of the emission.

\begin{tabular}{|c|c|c|}
\hline & $\begin{array}{c}\text { (a) UV excitation } \\
\text { Center } S_{a}\end{array}$ & Center $\mathrm{S}_{\mathrm{b}}$ \\
\hline $\mathrm{E}_{\text {center }}\left(\mathrm{cm}^{-1}\right)$ & $22400 \pm 180$ & $23900 \pm 130$ \\
\hline Height (arb. units) & $0.18 \pm 0.02$ & $0.06 \pm 0.03$ \\
\hline FWHM $\left(\mathrm{cm}^{-1}\right)$ & $3100 \pm 180$ & $2100 \pm 300$ \\
\hline$\Delta R(\AA)$ & $-0.13 \pm 0.01$ & $-0.08 \pm 0.01$ \\
\hline \multirow[t]{2}{*}{$\underline{\mathrm{E}_{\mathrm{ZPL}}\left(\mathrm{cm}^{-1}\right)}$} & $27000 \pm 3000$ & $26000 \pm 7000$ \\
\hline & $\begin{array}{l}\text { (b) UV-IR excitation } \\
\text { Center } S_{a}\end{array}$ & Center $S_{b}$ \\
\hline$\overline{\mathrm{E}_{\text {center }}\left(\mathrm{cm}^{-1}\right)}$ & $22300 \pm 200$ & $24000 \pm 140$ \\
\hline Height (arb. units) & $0.36 \pm 0.04$ & $0.20 \pm 0.06$ \\
\hline FWHM $\left(\mathrm{cm}^{-1}\right)$ & $3000 \pm 200$ & $2300 \pm 170$ \\
\hline$\Delta R(\AA)$ & $-0.12 \pm 0.01$ & $-0.09 \pm 0.01$ \\
\hline $\mathrm{E}_{\mathrm{ZPL}}\left(\mathrm{cm}^{-1}\right)$ & $27000 \pm 4000$ & $27000 \pm 4000$ \\
\hline
\end{tabular}

Sec. III E will suggest that previous pulses of IR excitation have heated the local environment of the ITEs, so this spectrum is equivalent to a UV emission spectrum at a higher temperature than $8 \mathrm{~K}$, and the temperature depends on the IR excitation energy density.

The emission band widths are very different for the two charge-compensation centers of $\mathrm{NaMgF}_{3}: \mathrm{Yb}^{2+}$. The two centers must have significantly different charge-compensation geometries. The emission band widths for $\mathrm{NaMgF}_{3}: \mathrm{Yb}^{2+}$ are less than in $\mathrm{CaF}_{2}: \mathrm{Yb}^{2+}$, but the emission bands for both systems are much wider than the $4 f^{13} 5 d^{1}$ absorption transitions-the lowest-energy $4 f^{13} 5 d^{1}$ absorption transition is $800 \mathrm{~cm}^{-1}$ wide in $\mathrm{CaF}_{2}: \mathrm{Yb}^{2+}$ (from Ref. 16). The absorption widths are difficult to measure in $\mathrm{NaMgF}_{3}: \mathrm{Yb}^{2+}$ due to multiple origins at similar energies. ${ }^{5}$

A transition with a large change in bond length will require the system to nonradiatively relax to its equilibrium configuration, so the emission is vibronically broadened. Bond length changes may be estimated for both centers from the emission bandwidths $\Gamma$ in Table I as

$$
\Delta R=\frac{\sqrt{\hbar}}{\sqrt{5.57 \pi c}} \frac{\Gamma}{\sqrt{\mu n_{\operatorname{lig}} \nu^{3}}},
$$

where $\mu$ is the effective ligand mass, $n_{\text {lig }}$ is the coordination number, and $v$ is the effective vibrational frequency. ${ }^{17}$

The effective phonon frequency used is $325 \mathrm{~cm}^{-1}$, which is estimated for $\mathrm{CaF}_{2}: \mathrm{Yb}^{2+}$ in Ref. 6 and should allow comparison between fluoride systems. The $\mathrm{Yb}^{2+}$ center in $\mathrm{NaMgF}_{3}$ is assumed to have a coordination number of $12 .{ }^{12}$ The calculation gives a $0.13 \AA$ decrease in bond length when the system is in the ITE of center $S_{a}$ and a decrease of $0.08 \AA$ when the system is in the ITE of center $S_{b}$. These values are indications only as the phonon frequency and coordination number are approximate.

The phonon frequency and coordination number are presumably very similar for the two centers so comparison between the bond length change for centers $S_{a}$ and $S_{b}$ can be made. $S_{a}$ has a greater bond length change than $S_{b}$. A greater change in bond length can occur when the bond is longer, so the bond lengths indicate that $S_{a}$ represents a $\mathrm{Yb}^{2+}$ center which is further from surrounding ligands than $\mathrm{S}_{\mathrm{b}}$.

Regardless of which center we look at, when IR excitation is applied the higher excitonic state becomes more influential in emission, so to compare the configuration of the ITE states we can look at the bond length change in Table I after UV excitation or after UV+IR excitation. The bond length change is very similar for center $S_{a}$ after $U V$ or $U V+I R$ excitation. In both centers the ITE configuration coordinate is very similar for the two ITE states.

A transition with a large shift in configuration will cause a shift in the energy of radiative emission, as some of the transition energy will be emitted vibrationally. When the vibrational frequencies of the initial and final states are assumed to be the same, the shift in the center of the emission energy $E_{\text {center }}$ from the zero phonon line energy $E_{\text {zpl }}$ can also be estimated from the emission band width, as

$$
E_{\mathrm{ZPL}}=E_{\text {center }}-\left(\left(\frac{\Gamma}{2.36 v}\right)^{2}-\frac{1}{2}\right) v .
$$

A detailed derivation of Eqs. (1) and (2) is found in Ref. 17. The approximate ZPL positions for both centers of $\mathrm{NaMgF}_{3}: \mathrm{Yb}^{2+}$ are listed in Table I.

\section{B. Two-dimensional spectroscopy}

Two-dimensional representations of the emission enhancement are built up by scanning across a range of IR frequencies with the FEL $(5-12 \mu \mathrm{m})$ and recording the transient intensity from $0-800 \mu$ s after the IR excitation at each IR frequency. In Fig. 3 the sample has been excited with a $300 \mathrm{~nm}$ UV pulse, creating ITEs. The subsequent emission is from two ITE energy levels, shown in Fig. 1. The difference in radiative rates between these energy levels means the population ratio between the states can be inferred from the dynamic behavior of the emission. When the temperature of the sample is at $8 \mathrm{~K}$ most of the population is initially in the ITE ground state (with a lifetime of the order of $8 \mathrm{~ms}$ ). At $t=0$ an IR pulse excites the ITE into states with a shorter radiative lifetime (of the order of $300 \mu \mathrm{s}$ ), which generates an emission enhancement via the population redistribution. When the temperature is increased to $40 \mathrm{~K}$, the higher ITE state becomes thermally populated leaving fewer excitons available for IR excitation directly from the ground state. Figure 3 shows the emission monitored at the center of the band in Fig. 2(a), which is a mixture of emission from centers $S_{a}$ and $S_{b}$. Figure 4 shows the SSTPE IR excitation spectrum for both centers by monitoring emission at the two energies indicated in Fig. 2(b).

The photoluminescence enhancement was studied as the UV excitation energy density was reduced from 70 to $5 \mathrm{~mJ} \mathrm{~cm}^{-2}$. After intensity normalizing the overall magnitude of the transient we see that the IR induced photoluminescence enhancement is independent of the UV excitation energy density. Since the enhancement doesn't depend on the UV energy density, the population redistribution between ITE energy levels caused by the IR excitation doesn't depend on the number of oscillators in ITE states before the IR excitation. 


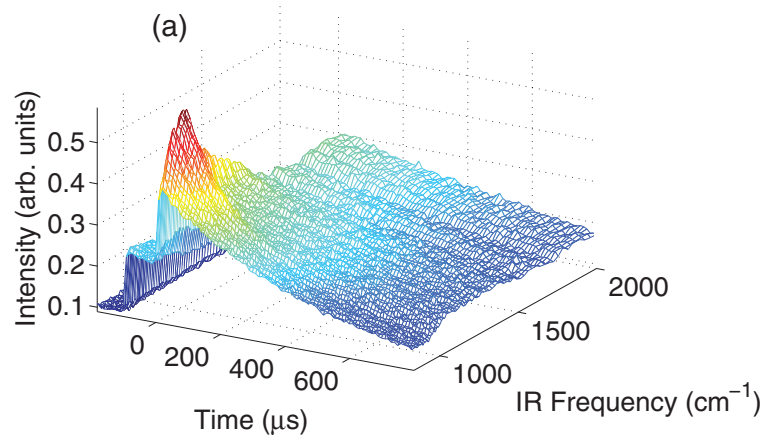

(b)

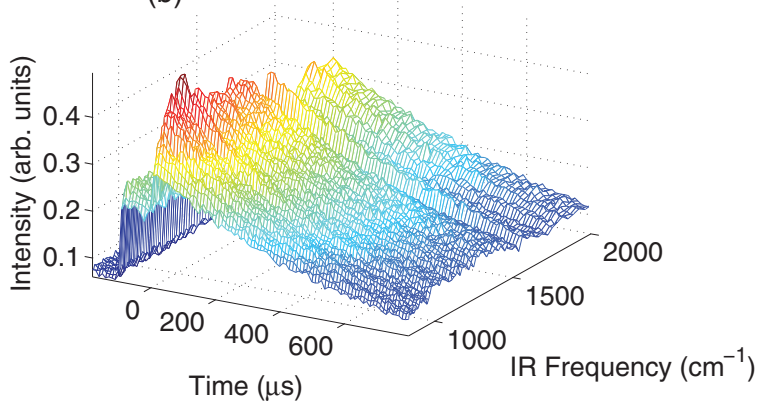

FIG. 3. (Color online) Time evolution of the SSTPE excitation spectrum of $\mathrm{NaMgF}_{3}: \mathrm{Yb}^{2+}$ subsequent to excitation with a $300 \mathrm{~nm}$ prepulse and IR excitation at $0 \mathrm{~s}$. Emission is monitored at $23300 \mathrm{~cm}^{-1}$ (430 nm). (a) $8 \mathrm{~K}$ and (b) $40 \mathrm{~K}$. The peak centered at $950 \mathrm{~cm}^{-1}$ is from intraexcitonic transitions.

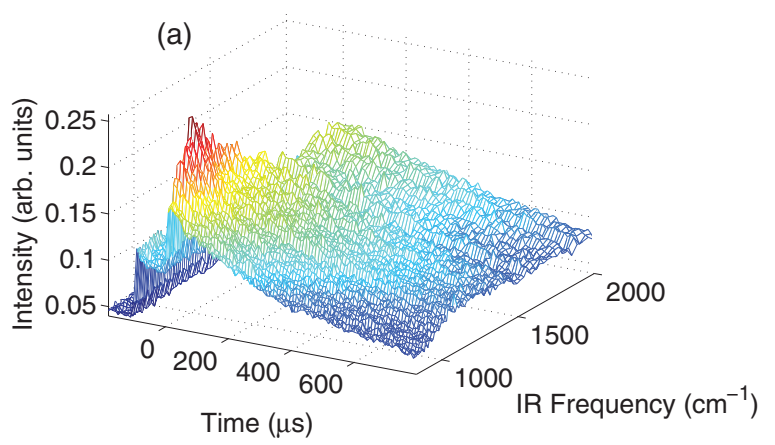

(b)

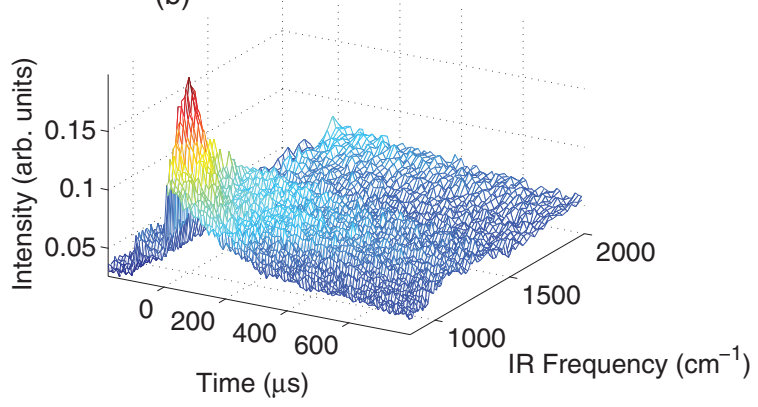

FIG. 4. (Color online) $8 \mathrm{~K}$ center-selective excitation spectra of $\mathrm{NaMgF}_{3}: \mathrm{Yb}^{2+}$ subsequent to excitation with a $300 \mathrm{~nm}$ prepulse at a temperature of $8 \mathrm{~K}$ and IR excitation at $0 \mathrm{~s}$. (a) Emission at $21000 \mathrm{~cm}^{-1}$ (emission 1 in Fig. 2) shows the dynamics of center $\mathrm{S}_{\mathrm{a}}$. (b) Emission at $25000 \mathrm{~cm}^{-1}$ (emission 2 in Fig. 2) shows the dynamics of center $S_{b}$.

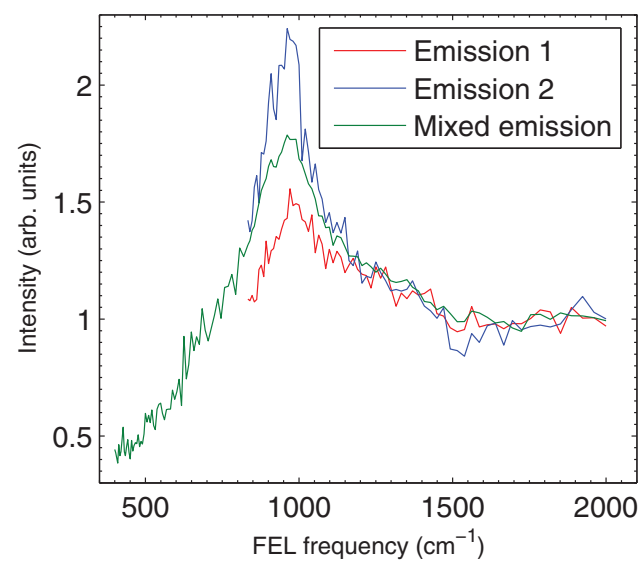

FIG. 5. (Color online) Emission of $\mathrm{NaMgF}_{3}: \mathrm{Yb}^{2+}$ shown in Fig. 4 integrated over the first $50 \mu$ s after the IR excitation and monitored in the middle and at either side of the emission band in Fig. 2. The emission at $430 \mathrm{~nm}$ has been separately measured for a lower energy region (from 500 to $900 \mathrm{~cm}^{-1}$ ) and normalized. The excitation peak indicates the transition between the excited ITE energy levels.

\section{Intraexcitonic absorption}

The excitation spectrum of the IR enhancement from both centers of $\mathrm{NaMgF}_{3}: \mathrm{Yb}^{2+}$ shows a distinct peak at $950 \mathrm{~cm}^{-1}$. The excitation spectrum (Fig. 5) is constructed by integrating the emission in Figs. 3 and 4 over the first $50 \mu$ s after the IR excitation. The reason for not integrating over the whole time range is that Fig. 5 illustrates the emission from the population that is excited by the IR beam to state 3 , and this population decays nonradiatively to state 2 within $100 \mu$ s. The decay time of $100 \mu \mathrm{s}$ is the nonradiative decay from states 3 to 2 and is not directly related to the radiative decay from states 2 and 3 . After several hundred microseconds the system will reach thermal equilibrium and most of the population will be in state 2 . The proportion of ITEs in state 3 compared to 2 will be fixed and depend on the temperature of the system.

Two separate excitation scans monitored at the emission peak $23300 \mathrm{~cm}^{-1}$ (430 nm)—one from 500 to $900 \mathrm{~cm}^{-1}$ and another from 830 to $2000 \mathrm{~cm}^{-1}$-are plotted together in Fig. 5 . The curves are all normalized so that the emission intensity at $2000 \mathrm{~cm}^{-1}$ is unity. The peak observed in these excitation spectra is interpreted as due to a resonant absorption between internal energy levels of the ITE. Such an upper level of the ITE is shown as level 4 in Fig 1.

The temporal analysis in Sec. III E allows us to confidently say the excitation peak at approximately $950 \mathrm{~cm}^{-1}$ in Fig. 5 shows the FEL is being resonantly absorbed into the higher exciton state, indicating the existence of excited ITE states $950 \mathrm{~cm}^{-1}$ above the ITE ground state.

\section{Trap liberation}

At some values of the IR frequency if we look at the intensity well after the population has reached thermal equilibrium (hundreds of microseconds after the IR excitation) the intensity of the emission is greater than before the IR pulse, which indicates that there is some mechanism providing additional oscillators into the radiative ITE levels. Fluoride systems show evidence of forming electron traps, so we 


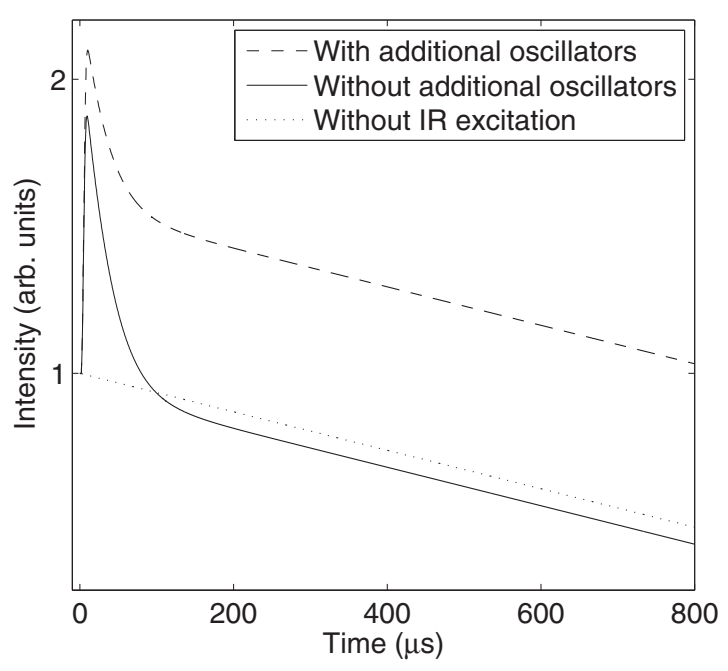

FIG. 6. Illustration of how the addition of extra oscillators via a trap liberation process changes the decay profile of the emission intensity of $\mathrm{NaMgF}_{3}: \mathrm{Yb}^{2+}$ after IR excitation.

postulate that the IR pulse is liberating electron traps to provide extra population to the ITE states. The UV excitation promotes electrons to the conduction band where they may become trapped, the population of trapped electrons is maintained over the experiment.

Traps occur in all optical materials and are difficult to characterize. They often come from growth impurities so they are not able to be replicated easily. This analysis treats the traps very generally, so emission related to traps is able to be differentiated from the optical mechanisms of ITE states.

If electron traps were not present we would expect the intensity of the emission to be lower than the intensity extrapolated by the population continuing to radiate from the ITE states at a constant rate. The IR excitation should redistribute population to an ITE state that decays faster, causing more population to leave the ITE system. There would be less population left in the system to radiate, so the emission intensity would be lower.

A simple model shown in Fig. 6 indicates the expected emission dynamics with and without additional oscillators. The expected difference in emission intensity can be seen by comparing the dotted and solid lines in Fig. 6. The dotted line is the intensity of emission if there is no redistribution of ITE population, so the oscillators continue to radiate from the ITE states at a constant rate. The solid line is the expected emission when the IR excitation redistributes population to an ITE state that decays faster without electron traps present. It would be expected that the intensity several hundred $\mu \mathrm{s}$ after the IR excitation to be equal to the intensity just before the IR excitation minus the number of photons emitted from the excitonic states since the IR excitation. We note that this increased emission occurs only at some IR frequencies, which is interpreted as the trap excitation process having a spectral dependence.

Figure 6 shows the general shape of the transients recorded when ITEs in $\mathrm{NaMgF}_{3}: \mathrm{Yb}^{2+}$ are excited with an IR beam. There is an initial increase in intensity, as population is now radiating from level 3 , which has a higher radiative rate than level 2. The intensity then decays exponentially. The lifetime of this initial decay indicates the rate from level 3 to level 2. After about $200 \mu$ s the system is in thermal equilibrium. There is population radiating from states 2 and 3 in fixed proportions determined by the temperature of the system.

Instead of the emission intensity after thermal equilibrium being lower than we would expect without IR excitation, population liberated from electron traps by the IR excitation creates ITEs, which increase the emission intensity at $800 \mu \mathrm{s}$ after the IR excitation above the initial intensity at some IR frequencies. The dashed line in Fig. 6 shows the scale of this increased emission intensity. To account for the increased emission intensity the IR excitation must introduce additional oscillators into the ITE system.

$$
n_{\text {trap }}=n_{\mathrm{f}}-n_{\mathrm{i}}+n_{\text {photons }} .
$$

Equation (3) is stating that the ITE population added from electron traps over some period (given by $n_{\text {trap }}$ ) is equal to the difference in ITE population over that time period $\left(n_{\mathrm{f}}-n_{\mathrm{i}}\right)$ plus the ITE population that has radiated in that time (called $\left.n_{\text {photons }}\right)$.

The number of photons emitted from the excitonic states in this time period will be $n_{\text {photons }}=\frac{1}{\tau} \int_{t_{\mathrm{f}}}^{t_{\mathrm{f}}} n(t) d t$, where $\tau$ is the lifetime of the emission decay before the IR pulse. By substituting $I=\frac{n}{\tau}$ for the instantaneous populations, we can derive an equation to calculate the number of oscillators added to the system (relative to the pre-IR ITE oscillator number) from intensity transients like the ones in Fig. 6. This equation is Eq. (4).

The calculation assumes that the temperature (and so the relative population of the ITE states 2 and 3 ) is the same before and several hundred microseconds after the IR pulse as the center heating is a cumulative effect over many IR pulses. Some additional population is lost from the nonradiative decay from the upper ITE levels $W_{41}$, but it is negligible compared to the radiative loss.

The amount of ITEs that come from the liberated electron traps compared to the ITE population just before the IR excitation is given by the following equation:

$$
\frac{n_{\text {trap }}}{n_{\mathrm{i}}}=\frac{I_{\mathrm{f}}}{I_{\mathrm{i}}}-1+\frac{1}{\tau} \int_{t_{\mathrm{i}}}^{t_{\mathrm{f}}} \frac{I}{I_{\mathrm{i}}} d t,
$$

where $\tau$ is the lifetime of the emission decay before the IR pulse. The fraction calculated from Eq. (4) is shown in Fig. 7. All three emission frequencies have similar trap liberation spectra so the trap distribution must be similar at both centers. Looking at the emission intensity across IR frequency at $800 \mu \mathrm{s}$ in Fig. 4, there is the same rise at an IR excitation energy of $800 \mathrm{~cm}^{-1}$ as in Fig. 7.

The amount of ITEs that come from the liberated electron traps varies enormously with IR excitation energy. The activation energy of the traps is clearly seen in the steep increase around $800 \mathrm{~cm}^{-1}$. The trap liberation spectrum of $\mathrm{NaMgF}_{3}: \mathrm{Yb}^{2+}$ is similar to that of $\mathrm{CaF}_{2}: \mathrm{Yb}^{2+}$ (Ref. 6) but the traps are deeper. Figure 7 indicates the traps in $\mathrm{NaMgF}_{3}: \mathrm{Yb}^{2+}$ have an approximate depth of $800 \mathrm{~cm}^{-1}$ compared to $400 \mathrm{~cm}^{-1}$ in $\mathrm{CaF}_{2}$ and $\mathrm{SrF}_{2} \cdot{ }^{18}$

At an IR excitation energy above $800 \mathrm{~cm}^{-1}$ the number of ITEs created from trap liberation is almost equal the number 


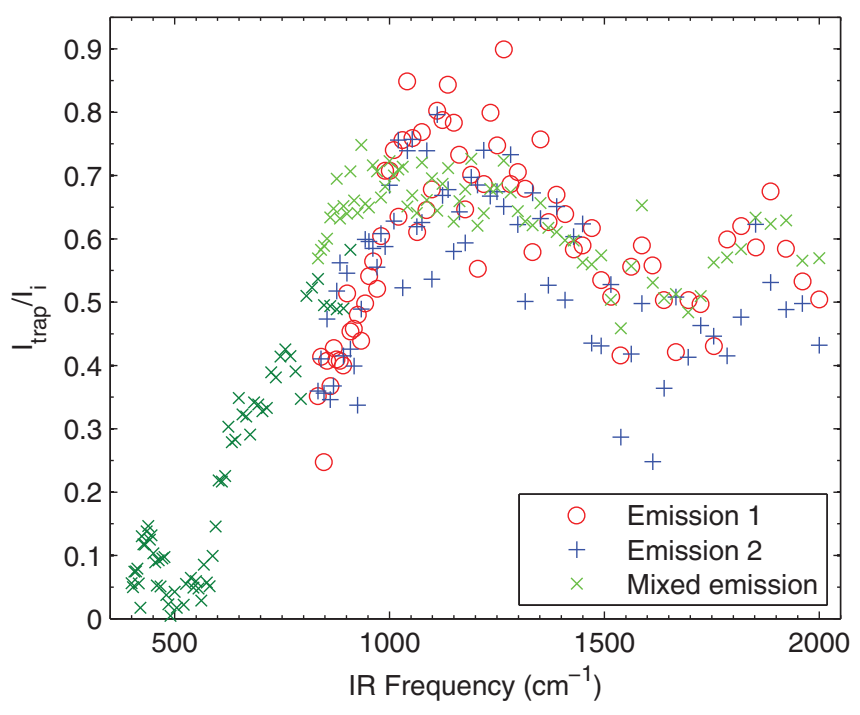

FIG. 7. (Color online) The proportion of ITE population that comes from liberated trap population compared to the population in ITE states before IR excitation. This proportion is calculated from Eq. (4) for three emission energies. The green points are from signal monitored in the middle of the emission band at $23300 \mathrm{~cm}^{-1}$. The emission at $23300 \mathrm{~cm}^{-1}$ has been separately measured for a lower energy region (from 500 to $900 \mathrm{~cm}^{-1}$ ) and normalized.

created directly by UV excitation. There are clearly many electron traps in $\mathrm{NaMgF}_{3}$ and traps play an important role in the system's luminescence.

The proportion of ITEs created from the IR excitation liberating traps decreases rapidly with temperature. Applying Eq. (4) to transients at different temperatures shows the amount of trapped electrons liberated is zero by $20 \mathrm{~K}$, so the electrons in traps are made inaccessible to the IR excitation by a small amount of thermal energy. The electrons are unlikely to be liberated by the thermal energy at $20 \mathrm{~K}$ as the trap depth is too large at around $800 \mathrm{~cm}^{-1}$.

\section{E. Analysis of emission dynamics}

The temporal transients are modelled with a system of rate equations to represent the multilevel exciton and trap liberation. In the model, the UV pulse generates the exciton states 2 and 3 as well as free carriers in the conduction band, which subsequently fall into shallow traps. The IR pulse causes intraexcitonic transitions and liberates carriers from traps, some portion of which ultimately populate the exciton ground state.

The energy levels shown in Fig. 8 are:

1. the ground state of divalent ytterbium;

2. the ground state of the impurity trapped exciton, with radiative rate $A_{21}$;

3. a state of the impurity trapped exciton with, radiative rate $A_{31}>A_{21}$;

4. a higher-energy exciton state; and

5. the conduction band, populated by electron trap liberation.

There are two processes being modeled. The first is the IR pulse exciting excitons from the ground exciton state (2) to a higher exciton state (4) where they decay to the second

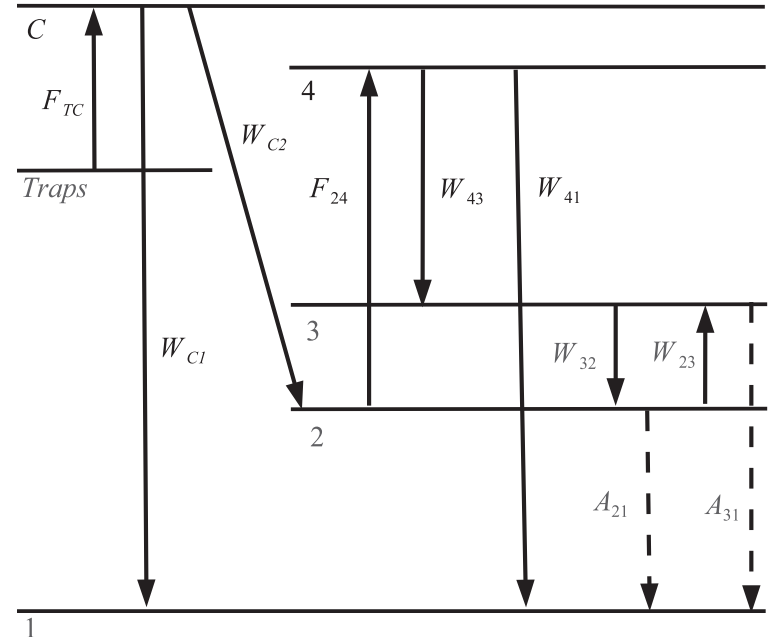

FIG. 8. The energy level transitions involved in the dynamic behavior of $\mathrm{NaMgF}_{3}: \mathrm{Yb}^{2+}$ after IR excitation. The transitions are labeled with the rates used in Eqs. (5)-(9). $W$ terms represent rates of nonradiative transitions. $F$ terms are mechanisms driven by the IR pulse, which are the population transfer from the lower to higher excitonic states $\left(F_{24}\right)$ and electrons released from traps $\left(F_{\mathrm{TC}}\right) . A_{21}$ and $A_{31}$ are the radiative rates from the ITE levels.

exciton state (3) and radiatively decay to the ytterbium ground state (1). The second process is the IR pulse liberating electrons from trap states to the conduction band $(C)$ where they recombine with ytterbium ions to form impurity-trapped excitons in the lower exciton state (2) which radiates to the ytterbium ground state (1). The change in population of the above states are modeled with the following rate equations:

$$
\begin{aligned}
& \frac{d N_{1}}{d t}=A_{21} N_{2}+A_{31} N_{3}+W_{41} N_{4}+W_{\mathrm{C} 1} N_{\mathrm{C}}, \\
& \frac{d N_{2}}{d t}=-\left(A_{21}+W_{23}+F_{24}\right) N_{2}+W_{32} N_{3}+W_{\mathrm{C} 2} N_{\mathrm{C}}, \\
& \frac{d N_{3}}{d t}=W_{23} N_{2}-\left(A_{31}+W_{32}\right) N_{3}+W_{43} N_{4}, \\
& \frac{d N_{4}}{d t}=F_{24} N_{2}-\left(W_{41}+W_{43}\right) N_{4}, \\
& \frac{d N_{\mathrm{C}}}{d t}=F_{\mathrm{TC}}-\left(W_{\mathrm{C} 1}+W_{\mathrm{C} 2}\right) N_{\mathrm{C}} .
\end{aligned}
$$

The initial population distribution is at thermal equilibrium, and the assigned temperature is the same as at several hundred $\mu$ s after the IR excitation. The pumping term $F_{24}$ is the rate of population transfer from the lower to higher excitonic states. $F_{\mathrm{TC}}$ is proportional to the number of electrons released from traps. $W_{\mathrm{C} 2}$ is the rate at which population that has been added to the system from liberated electron traps (determined by $F_{\mathrm{TC}}$ ) recombines with ytterbium ions to form ITEs in their ground state. A better fit to the experimental data was achieved when the parameter $W_{\mathrm{C} 3}$ representing ITEs forming in their excited state was zero. $W_{\mathrm{C} 2}$ represents electron recombination to ITEs, and the nonradiative rates between the ITE states 2 and 3 control the distribution of these new oscillators within the ITE levels. $W_{43}$ is the nonradiative rate between higher excitonic states and the second excitonic state ( 3 on Fig. 8 ). $W_{41}$ and 
$W_{\mathrm{C} 1}$ are rates of population loss from the higher excitonic states and the conduction band respectively to the ground state. $A_{21}$ and $A_{31}$ are the radiative rates from the exciton levels.

The population in the higher excitonic state 4 that does not transition to the upper ITE state is modeled as only going to the ground state in a rate $W_{41}$. The model can be modified so this population goes instead to the lowest ITE state in a rate $W_{42}$. If $W_{42}$ is used instead of $W_{41}$, more oscillators remain in the ITE system after the IR excitation and to balance this the rate of oscillators entering the system via trap liberation $F_{\mathrm{TC}}$ is less. It is still necessary to have trap liberation to explain the intensity hundreds of microseconds after IR excitation. Since there is no way of determining the ratio between the rates $W_{41}$ and $W_{42}$, in the model presented here $W_{42}$ is assumed to be zero which gives a better fit than assuming $W_{41}$ is zero.

To ensure thermal equilibrium, the nonradiative rates between the exciton states 2 and 3 are related by a Boltzmann factor:

$$
W_{23}=W_{32} \frac{g_{3}}{g_{2}} \exp \left(\frac{-\Delta E_{23}}{k T}\right),
$$

where $g_{2}$ and $g_{3}$ are the energy level degeneracies, $\Delta E_{23}$ is the energy gap separating the exciton energy levels 2 and $3, T$ is temperature, and $k$ is Boltzmann's constant. The level degeneracies are set to $g_{2}=1$ and $g_{3}=3$ because, as in the case of the systems analyzed by Moine et al., ${ }^{16}$ the structure of $\mathrm{NaMgF}_{3}$ can be approximated as octahedral. In an octahedral system the exciton energy levels will be the result of coupling a doubly degenerate delocalized ${ }^{2} S$ state with a doubly degenerate $4 f^{13}$ state, which will give a singlet and triplet state.

The nonradiative relaxation rates from the exciton energy levels 2 and 3 to the ground state 1 have not been taken into account. The emission of $\mathrm{NaMgF}_{3}: \mathrm{Yb}^{2+}$ under UV excitation is not quenched until temperatures much higher than $50 \mathrm{~K}^{5}$, so the nonradiative rates are negligible in comparison to the radiative rates $A_{21}$ and $A_{31}$ at temperatures below $50 \mathrm{~K}$.

The radiative rates and the energy level gap between the excitonic states have been previously calculated from the temperature dependence of the total emission lifetime. The parameters in Table II were found by fitting the

TABLE II. Parameter values for modeling $\mathrm{NaMgF}_{3}: \mathrm{Yb}^{2+}$ emission. These rates are used throughout the analysis whereas other rates shown in Fig. 8 are varied to model different power, temperature, and excitation frequencies. The radiative rates and ITE energy level differences were calculated from the temperature dependence of the total emission lifetime of Lizzo et al. (Ref. 5).

\begin{tabular}{lcc}
\hline \hline & Center $\mathrm{S}_{\mathrm{a}}$ & Center $\mathrm{S}_{\mathrm{b}}$ \\
\hline$A_{21}\left(\mathrm{~s}^{-1}\right)$ & $132(7.6 \mathrm{~ms})$ & $113(8.8 \mathrm{~ms})$ \\
$A_{31}\left(\mathrm{~s}^{-1}\right)$ & $2066(480 \mu \mathrm{s})$ & $2817(350 \mu \mathrm{s})$ \\
$\Delta E_{23}\left(\mathrm{~cm}^{-1}\right)$ & 24 & 62 \\
$g_{2}$ & 1 & 1 \\
$g_{3}$ & 3 & 3 \\
$W_{\mathrm{C} 2}\left(\mathrm{~s}^{-1}\right)$ & $5.0 \times 10^{5}$ & $5.0 \times 10^{5}$ \\
\hline \hline
\end{tabular}

lifetime data recorded by Lizzo et al. ${ }^{5}$ to a two-level model following the method of Moine et al. and assuming the rate of thermalization between the radiating ITE levels is much faster than the radiative rates of the ITE levels. ${ }^{16}$

Note the radiative rates and energy level gaps are not the same as Lizzo et al.'s values ${ }^{5}$ because they chose the degeneracies of the energy levels as $g_{2}=g_{3}=1$ rather than $g_{3}=3, g_{2}=1$ as discussed above. The change in degeneracies only alters $A_{21}$ and $\Delta E_{23}$ by a few percent but the value of $A_{31}$ fitted with the new degeneracies is about a half of that calculated by Lizzo et al. If the degeneracies are left at Lizzo's values, the temperature predicted by the model only increases by $5 \mathrm{~K}$ when the indicated temperature of the sample is increased by $23 \mathrm{~K}$. If we use $g_{3}=3, g_{2}=1$ the model temperature changes more reasonably, as will be discussed in Sec. III G.

The energy difference between the ITE states is less for center $S_{a}$ than it is for center $S_{b}$, as shown in Table II. Given the variation in energy difference, if the temperature of the system is increased from $8 \mathrm{~K}$, the intensity of emission from center $S_{a}$ should increase more than from center $S_{b}$. From Fig. 2, we see that when the system is excited with IR radiation the opposite occurs-emission from center $S_{b}$ increases more than emission from center $S_{a}$. This center-dependent mechanism and the varying lifetimes of the IR-induced emission enhancement indicates the IR pulse is having some effect other than simply heating the sample.

In the following analysis the parameters in Eqs. (5)-(9) (shown in Fig. 8) are used to simulate the emission enhancement as it varies with temperature, power, and IR frequency. In addition, IR excitation yields a modest heating related enhancement to the nonradiative rates. The parameter uncertainties $\sigma$ are estimated from the reduced $\chi^{2}$ of the fit of transients generated by the parameters to the data by the standard equation $\sigma=\sqrt{C \chi^{2}}$, where $C$ is the variance.

\section{F. IR fluence dependence}

The characteristics of the dynamics for a range of IR excitation energy densities are seen in Fig. 9. In all cases there is an initial emission enhancement associated with excitation to ITE level 3 and subsequent decay with a lifetime around $70 \mu \mathrm{s}$. Since this initial decay is faster than either of the radiative rates of the ITE energy levels (which are around $300 \mu \mathrm{s}$ and $8 \mathrm{~ms}$ ) it must be directly related to the predominantly nonradiative relaxation of ITE level 3 to $2\left(W_{32}\right)$. After about $200 \mu \mathrm{s}$, the levels are in thermal equilibrium. For the purposes of comparing the magnitude of enhancement, all of the transients presented in Figs. 9-11 have been normalized so that the emission intensity prior the IR pulse is the same.

Since the spectral deconvolution in Fig. 2 shows emission from center $S_{b}$ is less intense than from center $S_{a}$, the contribution of emission from center $S_{a}$ at emission 2 must be taken into account when analyzing the dynamics of center $\mathrm{S}_{\mathrm{b}}$. To model the IR-induced enhancement at emission 2 (400 nm) in Figs. 9-11 the intensity is calculated as $64 \%$ from center $\mathrm{S}_{\mathrm{b}}$ and $36 \%$ from center $\mathrm{S}_{\mathrm{a}}$. The intensity ratio between the centers may change with temperature and power, 

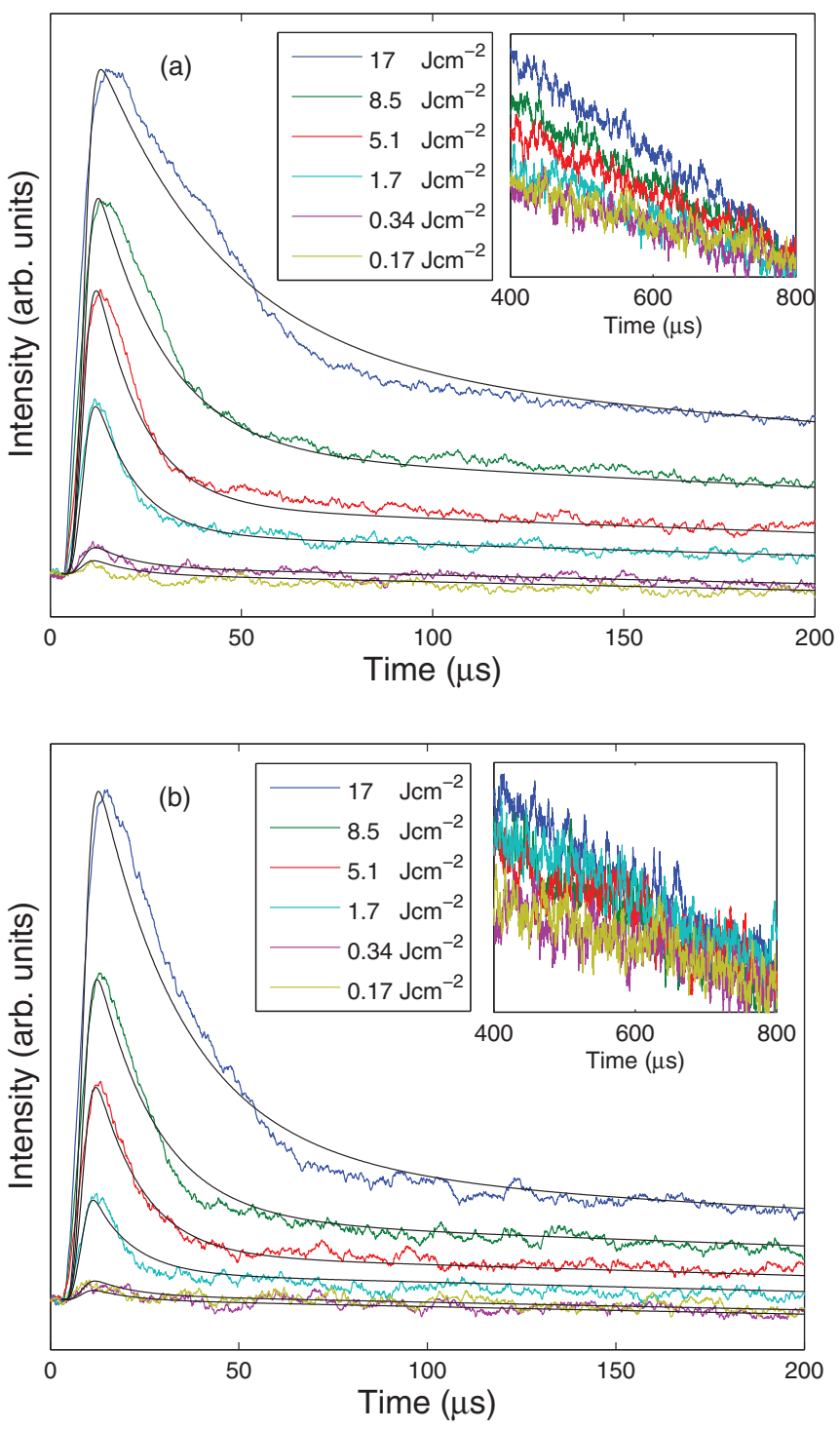

FIG. 9. (Color online) IR energy density-dependent dynamics of the IR enhancement of two centers of $\mathrm{NaMgF}_{3}: \mathrm{Yb}^{2+}$ already excited with a $300 \mathrm{~nm}$ prepulse at a indicated temperature of $5 \mathrm{~K}$. The IR excitation is $962 \mathrm{~cm}^{-1}$ at $0 \mathrm{~s}$. The black transients are calculated from the rate equation model with optimal parameters. The insets show the emission rate after thermal equilibrium is reached. (a) Emission monitored at $21000 \mathrm{~cm}^{-1}$ and entirely from center $\mathrm{S}_{\mathrm{a}}$. (b) Emission monitored at $25000 \mathrm{~cm}^{-1}$ and predominantly from center $S_{b}$.

so the parameters for center $S_{b}$ are less reliable than those from center $S_{a}$.

Figure 9 shows the variation of the photoluminescence enhancement as the time-averaged $962 \mathrm{~cm}^{-1}$ IR energy density is varied from $0.2-17 \mathrm{~J} \mathrm{~cm}^{-2}$. After more than about $200 \mu \mathrm{s}$ the decay can be described by a single exponential function with a lifetime that is proportional to the relative population in the higher and lower ITE states ( 2 and 3 ). The distribution of population between states 2 and 3 is directly related to temperature [via Eq. (10)] so the temperature of the center can be found from the measured radiative lifetime of the emission after $200 \mu \mathrm{s}$-assuming the radiative rates and energy level
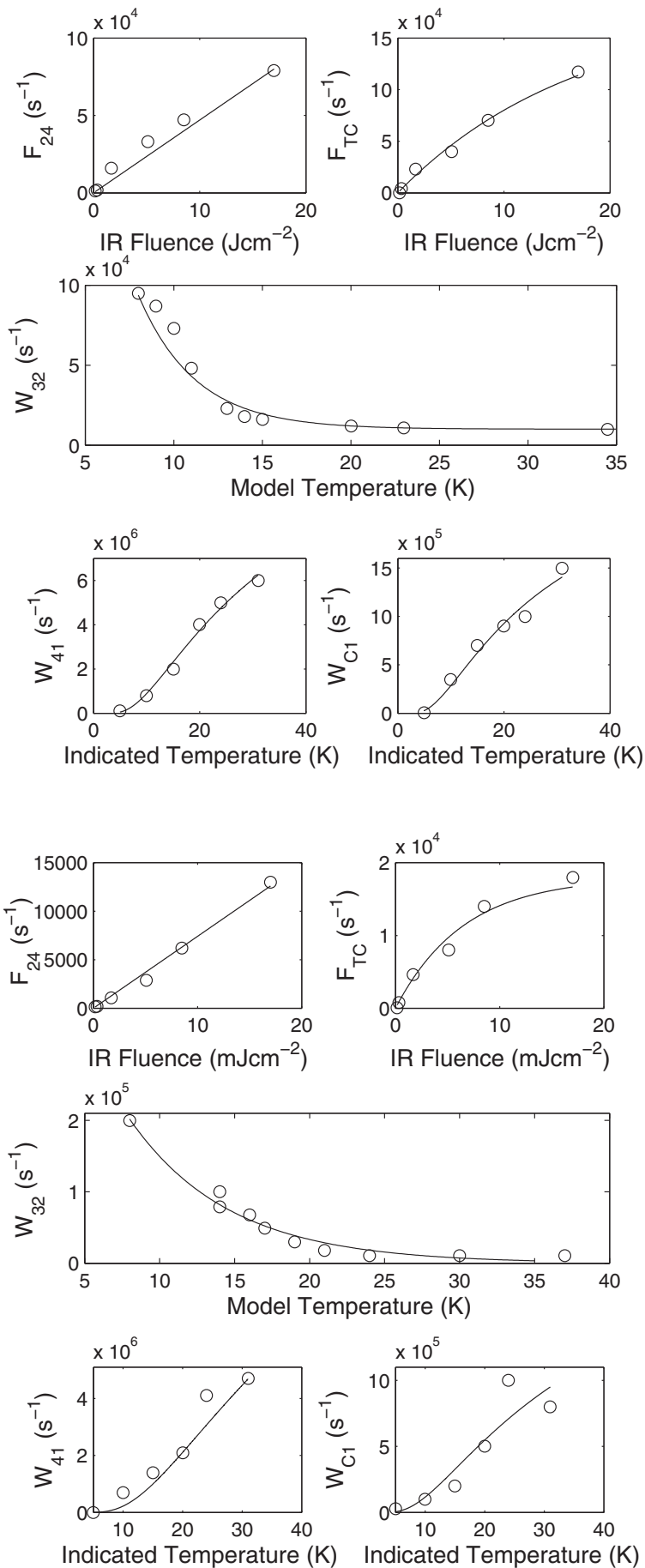

FIG. 10. The parameter values for the transients varying with IR fluence and temperature. The circles are the optimal values of the parameters used to model the transients in Figs. 9 and 11 and the lines are the fits to those values, described in the Eqs. (11)-(15).

difference calculated from temperature dependence of lifetime are correct. This calculated center temperature is called the model temperature and is distinct from the indicated temperature that is set for the whole crystal. Although the indicated temperature remains constant, the model temperature is found to increase with increasing IR energy density (see 

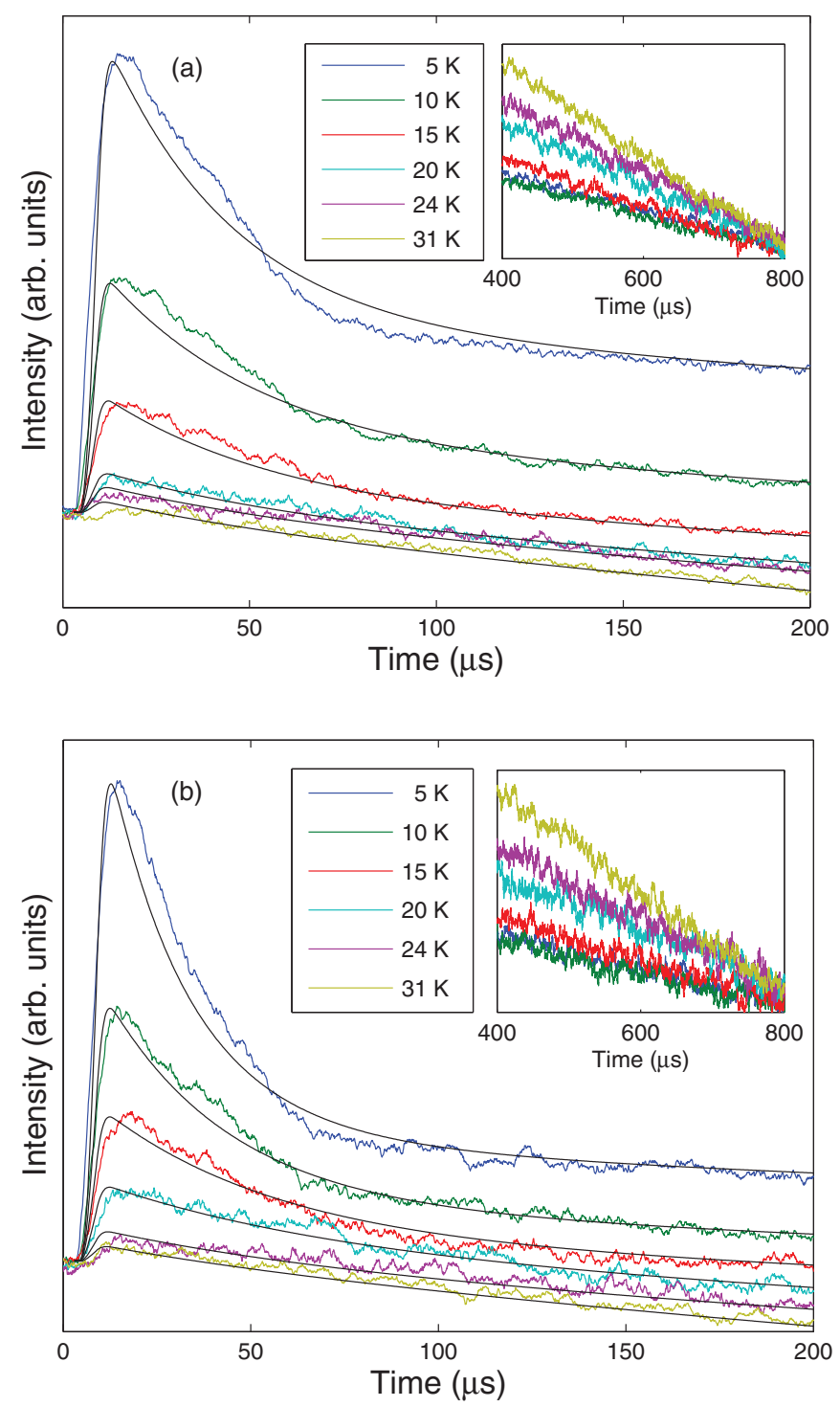

FIG. 11. (Color online) Temperature-dependent dynamics of the IR enhancement of two centers of $\mathrm{NaMgF}_{3}: \mathrm{Yb}^{2+}$ already excited with a $300 \mathrm{~nm}$ prepulse. The IR excitation is $17 \mathrm{~J} \mathrm{~cm}^{-2}$ and $962 \mathrm{~cm}^{-1}$ at $0 \mathrm{~s}$. The black transients are calculated from the rate equation model with optimal parameters. The insets show the emission rate after thermal equilibrium is reached. (a) Emission monitored at $21000 \mathrm{~cm}^{-1}$ and entirely from center $S_{a}$. (b) Emission monitored at $25000 \mathrm{~cm}^{-1}$ and predominantly from center $S_{b}$.

Table III) so the IR pulse must heat the sample. The change in temperature is different for the two centers.

It is important to bear in mind the parameters for center $S_{b}$ are determined by analyzing emission transients that are a mix

TABLE III. Comparison of the modeled temperatures used to calculate the emission dynamics for a range of IR energy densities (shown in Fig. 9). Uncertainties in model temperature are approximately $0.5 \mathrm{~K}$.

\begin{tabular}{lcccccc}
\hline \hline IR energy density $\left(\mathrm{J} \mathrm{cm}^{-2}\right)$ & 0.2 & 0.3 & 1.7 & 5.1 & 8.5 & 17 \\
Center $\mathrm{S}_{\mathrm{a}}$ model temperature $(\mathrm{K})$ & 8 & 8 & 9 & 10 & 11 & 13 \\
Center $\mathrm{S}_{\mathrm{b}}$ model temperature $(\mathrm{K})$ & 8 & 8 & 14 & 14 & 16 & 17 \\
\hline
\end{tabular}

of emission from both centers. If the ratio between the centers is changed slightly (from 64:36 to 54:46) the long time slopes of the transients at emission 2 can be accurately reproduced with the same model temperatures for center $S_{b}$ as center $S_{a}$. Therefore the difference in IR heating between the centers is probably due to uncertainty in the contributions of either center to the emission 2 transients rather than a physical difference in the heating.

The four rate equation parameters that vary with IR fluence are the rate the IR excitation promotes population from the ground ITE state to a higher ITE state 4, the number of electrons released from traps, the nonradiative rate from ITE level 3 to 2 , and the model temperature, which effects the nonradiative rate from level 2 to 3 .

The rate equation model was allowed to run on all the experimental transients where IR energy density was varied. Optimum values of the rate equation parameters were obtained from the fits with $\mathrm{F}_{24}, \mathrm{~F}_{\mathrm{TC}}$ and $\mathrm{W}_{32}$ being allowed to vary. The optimal values of the varied parameters are shown in Fig. 10. By considering a parameter dependence on IR energy density that makes physical sense a series of phenomenological equations were developed. They are Eqs. (11)-(13).

The parameter $F_{24}$ increases linearly with IR energy density, as expected since $F_{24}$ is a mechanism driven by the IR excitation.

$$
F_{24}=F_{24}^{m} \frac{P}{P_{\max }},
$$

where $P$ represents the time-averaged IR energy density. $P_{\max }$ is the maximum IR energy density, $17 \mathrm{~J} \mathrm{~cm}^{-2}$.

$F_{\mathrm{TC}}$ becomes saturated with IR energy density, indicating that the IR excitation creates close to the maximum amount of excitons from trap liberation even at low powers.

$$
F_{\mathrm{TC}}=F_{\mathrm{TC}}^{i}\left[1-\exp \left(-\xi_{\mathrm{TC}} \frac{P}{P_{\max }}\right)\right] .
$$

The emission decay in the first $100 \mu$ s after the IR pulse is noticeably faster as the IR energy density is decreased. To model this, the nonradiative rate between the higher and lower excitonic states $W_{32}$ decreases as power is increased. This is unusual in light of the increase in lattice temperature. It can be modelled in terms of the model temperature $T_{m}$ :

$$
W_{32}=W_{32}^{0} \exp \left(-\delta_{32} T_{m}\right)+c_{32} .
$$

Table IV lists the values used in Eqs. (11)-(13) to generate parameters for the rate equations used to calculate the black transients in Fig. 9. $\xi_{\mathrm{TC}}$ is greater for center $\mathrm{S}_{\mathrm{b}}$ which means that the rate at which saturation occurs is faster for the center $\mathrm{S}_{\mathrm{b}}$ configuration. There are many types of electron traps in $\mathrm{NaMgF}_{3}$ so it is possible that the electrons that create ITEs are liberated from different types of traps, or the configuration of center $\mathrm{S}_{\mathrm{b}}$ is such that more liberated electrons combine with center $S_{b}$ impurities as IR power is increased than at center $S_{a}$ impurities. To study the details of electron traps in $\mathrm{NaMgF}_{3}$ very precise temperature dependencies are needed and the details of the center configuration also require careful analysis.

$W_{32}^{0}$ is higher for center $S_{\mathrm{a}}$ than center $S_{\mathrm{b}}$, but both values are very large. The slow rate of thermal equilibrium between 
TABLE IV. The values for Eqs. (11)-(13) that were found by fitting the change optimal rate equation parameters with IR fluence in Fig. 10.

\begin{tabular}{lcc}
\hline \hline & Center $\mathrm{S}_{\mathrm{a}}$ & Center $\mathrm{S}_{\mathrm{b}}$ \\
\hline$F_{\mathrm{TC}}^{i}\left(\mathrm{~s}^{-1}\right)$ & $(1.80 \pm 0.01) \times 10^{5}$ & $(1.8 \pm 0.1) \times 10^{4}$ \\
$\xi_{\mathrm{TC}}$ & $1.00 \pm 0.08$ & $2.6 \pm 0.1$ \\
$W_{\mathrm{C} 1}\left(\mathrm{~s}^{-1}\right)$ & $(2.7 \pm 0.1) \times 10^{4}$ & $(2.5 \pm 0.1) \times 10^{3}$ \\
$F_{24}^{m}\left(\mathrm{~s}^{-1}\right)$ & $(8.00 \pm 0.04) \times 10^{4}$ & $(1.4 \pm 0.3) \times 10^{4}$ \\
$W_{41}\left(\mathrm{~s}^{-1}\right)$ & $(1.10 \pm 0.06) \times 10^{5}$ & $(2.5 \pm 0.2) \times 10^{3}$ \\
$W_{32}^{0}\left(\mathrm{~s}^{-1}\right)$ & $(1.0 \pm 0.3) \times 10^{6}$ & $(6.7 \pm 0.5) \times 10^{5}$ \\
$\delta_{32}\left(\mathrm{~K}^{-1}\right)$ & $0.31 \pm 0.04$ & $0.15 \pm 0.05$ \\
$c_{32}\left(\mathrm{~s}^{-1}\right)$ & $(1.0 \pm 0.4) \times 10^{4}$ & $(0 \pm 2) \times 10^{3}$ \\
\hline \hline
\end{tabular}

the two ITE levels could be because there are few phonons at the low energy between levels 2 and 3 .

\section{G. Temperature dependence}

As the temperature is increased there is less enhancement in emission, since there is more population in the upper ITE state and the IR pulse redistributes less population. In Fig. 11 the increase in temperature causes a decrease in initial enhancement and an increase in the slope after $200 \mu \mathrm{s}$.

Table V compares the temperature indicated in the experiment to the temperature used in the rate equation model to generate the black transients in Fig. 11. The model temperature is determined by the decay rate of the transient more than $200 \mu \mathrm{s}$ after the IR excitation. When the indicated crystal temperature is $4.9 \mathrm{~K}$, center $S_{\mathrm{b}}$ has a model temperature of $17 \mathrm{~K}$. This $12 \mathrm{~K}$ offset is consistent with the apparent strong IR absorption for this configuration. This absorption is characterised by a large rise in the model temperature with higher average IR energy density.

Equations (14) and (15) describe the trends in the rate equation parameters that vary with temperature. The rate of nonradiative loss from the higher excitonic levels $W_{41}$ increases with temperature. The thermal barrier is modeled with an Arrhenius function:

$$
W_{41}=W_{41}^{0} \exp \left(\frac{-\epsilon_{41}}{k T_{i}}\right),
$$

where $T_{i}$ represents the indicated temperature. $T_{i}$ is used rather than the model temperature because the conduction band will be at the temperature of the bulk crystal rather than the local temperature of the ITEs. Similarly there is nonradiative loss in the trap process $W_{\mathrm{C} 1}$.

$$
W_{\mathrm{C} 1}=W_{\mathrm{C} 1}^{0} \exp \left(\frac{-\epsilon_{\mathrm{C} 1}}{k T_{i}}\right) .
$$

TABLE V. Comparison of the modeled temperatures used to calculate the emission dynamics for a range of indicated temperatures (shown in Fig. 11). Uncertainties in model temperature are approximately $0.5 \mathrm{~K}$.

\begin{tabular}{lcccccc}
\hline \hline Indicated temperature (K) & 4.9 & 10 & 15 & 20 & 24 & 31 \\
Center $\mathrm{S}_{\mathrm{a}}$ model temperature (K) & 13 & 14 & 15 & 20 & 23 & 34 \\
Center $\mathrm{S}_{\mathrm{b}}$ model temperature (K) & 17 & 19 & 21 & 24 & 30 & 37 \\
\hline
\end{tabular}

TABLE VI. The values for Eqs. (14) and (15) that were found by fitting the change optimal rate equation parameters with temperature in figure Fig. 10.

\begin{tabular}{lcc}
\hline \hline & Center $\mathrm{S}_{\mathrm{a}}$ & Center $\mathrm{S}_{\mathrm{b}}$ \\
\hline$F_{\mathrm{TC}}\left(\mathrm{s}^{-1}\right)$ & $(1.2 \pm 0.1) \times 10^{5}$ & $(2.8 \pm 0.8) \times 10^{4}$ \\
$W_{\mathrm{C} 1}^{0}\left(\mathrm{~s}^{-1}\right)$ & $(3.00 \pm 0.05) \times 10^{6}$ & $(2.6 \pm 0.2) \times 10^{6}$ \\
$\epsilon_{\mathrm{C} 1}\left(\mathrm{~cm}^{-1}\right)$ & $17.0 \pm 0.2$ & $22.0 \pm 0.1$ \\
$F_{24}\left(\mathrm{~s}^{-1}\right)$ & $(7.90 \pm 0.04) \times 10^{4}$ & $(1.3 \pm 0.4) \times 10^{4}$ \\
$W_{41}^{0}\left(\mathrm{~s}^{-1}\right)$ & $(1.6 \pm 0.2) \times 10^{7}$ & $(2.0 \pm 0.3) \times 10^{7}$ \\
$\epsilon_{41}\left(\mathrm{~cm}^{-1}\right)$ & $20.0 \pm 0.3$ & $31 \pm 3$ \\
\hline \hline
\end{tabular}

Table VI lists the values found by fitting the Eqs. (14) and (15) to the optimal parameters for the rate equations, used to calculate the black transients in Fig. 11.

The parameter $F_{24}$ remains constant because the IR energy density is constant. The nonradiative rate between the excitonic states $W_{32}$ decreases with increasing model temperature, following the dependence on model temperature as in Eq. (13). This trend is consistent with the results obtained when varying the IR energy density illustrated in Eq. (13)

\section{CONCLUSION}

Measuring center-selective transient photoluminescence enhancement in multicenter $\mathrm{NaMgF}_{3}: \mathrm{Yb}^{2+}$ has increased the understanding of the dynamics of impurity-trapped excitons in $\mathrm{NaMgF}_{3}: \mathrm{Yb}^{2+}$. The experiments presented here have developed techniques for analyzing the interlevel transitions and dynamical behavior of insulator materials via two-photon spectroscopy. We have observed enhanced emission from impurity-trapped exciton states and electron trap liberation in both centers of $\mathrm{NaMgF}_{3}: \mathrm{Yb}^{2+}$. The temporal transients of the emission have been analyzed in terms of a multilevel rate equation model for both centers, and the dominant process that contributes to the dynamical behavior is intraexcitonic absorption and relaxation between three ITE levels. The exciton energy levels have been determined-two are only tens of wave numbers apart and one is $950 \mathrm{~cm}^{-1}$ from the exciton ground state. It is necessary to include electron trap liberation to explain the increase in emission intensity before and several hundred $\mu \mathrm{s}$ after IR excitation, and the electron trap depth is found to be approximately $800 \mathrm{~cm}^{-1}$. A moderate increase in local temperature around the ITE centers is inferred from the modeling. The detailed analysis of the temporal emission is relevant to understanding the dynamical behavior of impurity-trapped excitons in many scintillator materials.

\section{ACKNOWLEDGMENTS}

This work was supported by the Marsden fund of the Royal Society of New Zealand via Grant No. 09-UOC-080. We thank the Dutch FOM organization for providing FELIX beamtime and the FELIX staff for their assistance. R.B.H.C. and P.S.S. acknowledge support from the University of Canterbury. 
*Corresponding author: jon-paul.wells@ canterbury.ac.nz ${ }^{1}$ D. S. McClure and C. Pedrini, Phys. Rev. B 32, 8465 (1985).

${ }^{2}$ P. Dorenbos, J. Phys.: Condens. Matter 15, 2645 (2003).

${ }^{3}$ J. L. Pascual, J. Schamps, Z. Barandiarán, and L. Seijo, Phys. Rev. B 74, 104105 (2006).

${ }^{4}$ G. Sánchez-Sanz, L. Seijo, and Z. Barandiarán, J. Chem. Phys. 133, 114509 (2010).

${ }^{5}$ S. Lizzo, A. Meijerink, G. Dirksen, and G. Blasse, J. Phys. Chem. Solids 56, 959 (1995).

${ }^{6}$ M. F. Reid, P. S. Senanayake, Jon-Paul R. Wells, G. Berden, A. Meijerink, A. J. Salkeld, C.-K. Duan, and R. J. Reeves, Phys. Rev. B 84, 113110 (2011)

${ }^{7}$ P. S. Senanayake, J.-P. R. Wells, M. F. Reid, G. Berden, A. Meijerink, and R. J. Reeves, Appl. Phys. Lett. 100, 041902 (2012).

${ }^{8}$ N. J. M. Le Masson, A. J. J. Box, C. W. E. Van Eijk, C. Furetta, and J. P. Chaminade, Radiation Protection Dosimetry 100, 229 (2002).

${ }^{9}$ L. Struye and P. Leblans, US Patent No. US20010030302 (05/05 2001), http://www.freepatentsonline.com/y2010/0200741.html.
${ }^{10}$ C. Dotzler, G. V. M. Williams, U. Rieser, and A. Edgar, Appl. Phys. Lett. 91, 121910 (2007).

${ }^{11}$ C. J. Dotzler, A. Edgar, and G. V. M. Williams, US Patent No. US2010/0200741 (05/07 2007), http://www.freepatentsonline. com/y2010/0200741.html.

${ }^{12}$ E. Rönnebro, D. Noréus, K. Kadir, A. Reiser, and B. Bogdanovic, J. Alloys Compd. 299, 101 (2000).

${ }^{13}$ W. Zhang, H. Seo, B. Moon, S. Yi, and K. Jang, J. Alloys Compd. 374, 32 (2004).

${ }^{14}$ M. Grinberg, S. Mahlik, K. Wisniewski, and H. J. Seo, J. Phys.: Condens. Matter 23, 035404 (2011).

${ }^{15}$ J. Gâcon, A. Gros, H. Bill, and J. Wicky, J. Phys. Chem. Solids 42, 587 (1981).

${ }^{16}$ B. Moine, B. Courtois, and C. Pedrini, J. Phys. France 50, 2105 (1989).

${ }^{17}$ B. Henderson and G. F. Imbusch, Optical Spectroscopy of Inorganic Solids (Oxford University Press, Oxford, 1989).

${ }^{18}$ P. S. Senanayake, J. P. R. Wells, M. F. Reid, G. Berden, A. Meijerink, and R. J. Reeves, J. Lumin. 133, 81 (2013). 